\title{
Natural selection and immortality
}

\author{
Antoine Danchin
}

Received: 7 May 2008/Accepted: 5 August 2008/Published online: 22 August 2008

(C) The Author(s) 2008. This article is published with open access at Springerlink.com

\begin{abstract}
Genomes replicate while the host cells reproduce. I explore the reproduction/replication dialogue, based on a deep analysis of bacterial genomes, in relation to ageing. Making young structures from aged ones implies creating information. I revisit Information Theory, showing that the laws of physics permit de novo creation of information, provided an energy-dependent process preserving functional entities makes room for entities accumulating information. I identify explicit functions involved in the process and characterise some of their genes. I suggest that the energy source necessary to establish reproduction while replication is temporarily stopped could be the ubiquitous polyphosphates. Finally, I show that rather than maintain and repair the original individual, organisms tend to metamorphose into young ones, sometimes totally, sometimes progressively. This permits living systems to accumulate information over generations, but has the drawback, in multicellular organisms, to open the door for immortalisation, leading to cancer.
\end{abstract}

Keywords Persistent genes - Degradosome · Paleome · Metamorphosis · Isoaspartate

A. Danchin $(\bowtie)$

Genetics of Bacterial Genomes, CNRS URA2171,

Institut Pasteur, 28, rue du Docteur Roux,

Paris Cedex 15 75724, France

e-mail: antoine.danchin@normalesup.org

\section{Introduction}

All living organisms-bacteria included-age and eventually die. Because there is an enormous variation in the process (plants age slowly, for example) it is commonplace in ageing studies to consider ageing as a paradox that requires a deep explanation. This triggered a wealth of fascinating studies (and heated discussions) uncovering the variety of reasons and processes that account for ageing. In this context "immortality" is considered the norm. Why do we age, and how do we age (Kirkwood 2005)? This will not be the question I tackle here, but, rather, I will take here the exact opposite stance. Considering that ageing is the unavoidable consequence of straightforward physics - and this is indeed one of the major conclusions repeatedly reached by those who analysed ageing in-depth when they re-thought Orgel's error catastrophe (Orgel 1963) or Muller's ratchet (Muller 1932) theories-I observe, rather, that immortality (such as that we observe in cancer) raises a paradox. This paradox does not stem from the difficulty to account for an appropriate balance between errors and accuracy resulting in a sufficient leftover of correct replicating structures that can be retained over time (Orgel (Orgel 1970) and others (Kirkwood 1977), for example, have found a way out, but the window at $300 \mathrm{~K}$, the average temperature of life, is extremely narrow because of inherent thermal fluctuations). It derives from the remarkable but rarely pointed out everyday observation that "babies 
are born very young". Because they are made by aged organisms this requires an explanation, and it cannot be a trivial one.

Following a trend that has fallen in disuse (see the role of Schrödinger's book at the origin of molecular biology (Schrödinger 1945)), I proceed in a somewhat unusual way at the present stage of biological studies, placing life processes explicitly within the realm of Physics. I follow the most recent developments of Physics, that include information as a fifth category of Nature, which has to be superimposed on the four traditional ones, matter, energy, space and time (Steane 1998), making it an authentic independent category of Reality, not simply a derivative that would result from convolution of the properties of the four usual categories. Considering the model of the cell as a computer making computers, this novel trend fits remarkably well the separation between the genetic program, which replicates, and the machine that reads the program, which reproduces (Danchin 2009). As a matter of fact, while the genome of a daughter cell tends to be a replica of its parent, daughter cells are not a replica of their mother-cell (Rocha et al. 2003; Lindner et al. 2008), even in eukaryotic cells after mitosis (Fuentealba et al. 2008). I finally show, using an analysis of the genetic setup of single cell organisms, bacteria, how information can be concretely related to the four standard categories (matter, energy, space, and time) in explicit terms, identifying functions (with their cognate genes) and processes that may be at work in all living organisms, permitting some of them to manage information in such a way that creation of information is a central process of the formation of immortal time-sequences of organisms.

\section{A brief overview of ideas on ageing}

There are many definitions of ageing, mostly associated to the type of organism considered, and heavily dependent on emphasis placed on specific entities within the cell (the role of organelles, in particular, has been considerably stressed recently (Brunk and Terman 2002; Terman et al. 2007)). The question of ageing has essentially been restricted to the case of multicellular organisms where there is an obvious distinction to be made between the organism and its germ-line, as proposed by August Weismann many decades ago. For example, the disposable soma theory proposes that what we witness as ageing is essentially a side effect of longevity: reproduction of young organisms permits the organism to escape death of the soma, which rapidly remains a disposable leftover that may, under favourable circumstances display significant longevity (Kirkwood and Holliday 1979). A remark here: it is our usual anthropocentric view of Nature (Koyré 1973) which has led us to study ageing essentially in multicellular organisms, and more particularly in organisms where (some) individuals carry the germ line and propagate the species, while they become old and pass away. I try here to see this process as much more general-after all at least 50\% of the Earth's protoplasm is made of microbes-and investigate its relationship with the general constraint that operates on all living organisms, and that we know under the name Natural Selection. To this aim, I begin my investigation at the lowest level relevant for the study of life, the molecular level.

The core of the reflection on the very nature of ageing is based on a deep analysis of the consequences of the physico-chemical nature of replication. As already said, it comes from the idea that, unless specific maintenance and repair systems are organised in a proper non-trivial way, replication will inevitably accumulate errors until a moment when there might be an error catastrophe (Orgel 1963; Kirkwood 1977). A widely accepted view of ageing is summarised by Kirkwood: "Evolutionary considerations suggest ageing is caused not by active gene programming but by evolved limitations in somatic maintenance, resulting in a build-up of damage". And this implies that the core of ageing studies tries to understand the multiplicity of processes that cause damage to accumulate in cells and organisms (Kirkwood 2005; Terman et al. 2007). The general view, using a fairly loose concept of information (with even more fuzzy or naïve links with entropy, a topic that cannot be discussed here, but see (Danchin 2003)) is that in the balance between propagation of life and maintenance and repair, an energy-saving strategy of reduced error regulation will result in ageing of somatic cells (Kirkwood 1977, 2005). In this context, ageing is considered by many as somewhat irrelevant to the problem of perpetuation of the species, but, rather as essentially a problem faced by the individual organism, whether 
it reproduces or not. This reflection is particularly adapted to the case of individual organisms that have a role in the group but are not reproducing, such as is the case in social insects. It is naturally central to the reflection of individual human beings, who are doomed to face the burden of this inevitable process. The actual molecular processes at stake have, of course, to be uncovered, and much work has been devoted to this effort (see for recent reviews (Kirkwood 2005; Holliday 2006; Nystrom 2007; Partridge 2007; Terman et al. 2007; Rattan 2008)). One of the most fashionable view of the process is to relate maintenance to food supply, as longevity of many organisms (but certainly not all) appears to be related to food shortage or related processes (for a detailed review of the underlying hypotheses see (Kirkwood and Shanley 2005)).

Surprisingly, whereas there is a frequent tendency to see senescence as a fairly slow process, chemical analysis of proteins shows that many proteins tend to age extremely rapidly, even in the absence of the most commonly proposed stresses involved in ageing, such as reactive oxygen species (ROS) (Brunk and Terman 2002; Stroikin et al. 2005; Rattan 2008). Indeed, a major cause of protein ageing is spontaneous isomerisation of aspartate and asparagine, leading to the presence of isoaspartate residues (with concomitant deamidation in the case of the latter aminoacid) in the alpha carbon chain, distorting the protein backbone (Shimizu et al. 2005; Galletti et al. 2007). In many organisms, including humans, this process is counteracted by an energy-costly repair system involving $S$-adenosylmethionine-mediated methylation of isoaspartate and reversion to aspartate (Clarke 2003). The role of this repair system is so central that its deficiency leads in mammals to lethal diseases such as fatal progressive epilepsy (Yamamoto et al. 1998). A point worth noticing here: the very fact that the corresponding phenotype relates to the Central Nervous System, when neurons age essentially without reproducing, indicates that the process of repair, per se, has nothing to do with reproduction, creation of information and immortality. Despite the extreme importance of this process, not much studies have been devoted to its overall extent and influence on other general biological processes. In bacteria such as Escherichia coli, the isomerisation of aspartate is extremely rapid in some proteins. In ribosomal protein S11, for example, aspartate is converted into isoaspartate within minutes, suggesting (but by no means proving) that this isomerisation might belong to the normal function of the protein (David et al. 1999). In some proteins, this cyclisation results in a self-cleavage step essential for the function of the protein (Zarivach et al. 2008). During stationary phase, isoaspartate residues steadily accumulate, with a large proportion of proteins affected after $24 \mathrm{~h}$ of growth.

This process is consistent with recent experiments which show that contrary to a universally spread belief, bacteria, as all living organisms, would age even when other complex modifications such as ROSmediated modifications or protein glycation are not taken into account ((Nystrom 2003, 2007; Lindner et al. 2008), see also (Holliday 2006; Partridge 2007; Terman et al. 2007) for a general discussion of ageing in single cell and multicellular organisms). A further indication of the importance of this molecular process was found in organisms living in extreme environments. This isomerisation process being temperaturedependent, it was interesting to explore its importance in cold-living bacteria. As a matter of fact, asparagine residues are accumulating significantly in psychrophiles such as Pseudoalteromonas haloplanktis (which can grow fast at $0^{\circ} \mathrm{C}$ ) (Médigue et al. 2005) and Psychromonas ingrahamii (which can grow at temperatures as low as $-12^{\circ} \mathrm{C}$ ) (Riley et al. 2008), showing that this amino acid must have some physicochemical property important for protein structure (such as formation of asparagine ladders (Jenkins and Pickersgill 2001)) that is counterbalanced by its tendency to age. These observations imply that all organisms, including bacteria that reproduce by morphologically symmetric division, age (Stewart et al. 2005), and age fast.

More often than not however, it has been assumed that bacteria do not really age, at least when they are growing exponentially. While this was long suspected (Rocha et al. 2003; Lam et al. 2006; Watt et al. 2007), it is now firmly established that division is not symmetrical and that bacteria do age, even in these circumstances ((Stewart et al. 2005) and see discussion in (Stewart and Taddei 2005)). A consequence is that extant organisms are always composed with a significant proportion of aged structures, with the remarkable implication, rarely emphasised, that in the chain of descent, new organisms are always born from aged ones, sometimes as patchworks of aged 
and young structures. This may happen in various ways, sometimes with daughter cells somewhat similar to each other, especially when division looks superficially symmetrical (which it is not, in reality (Rocha et al. 2003; Stewart et al. 2005; Watt et al. 2007; Fuentealba et al. 2008; Lindner et al. 2008)), sometimes with a mother cell carrying most, if not all of the aged structures (Aguilaniu et al. 2003). This inevitable process opens the apparent paradox that aged biochemical structures (ribosomal proteins age, as just mentioned, and this may have enormous consequences as ribosomes are the central factories making proteins (Orgel 1963; Rattan 1996)) must direct synthesis of young ones.

\section{Reproduction versus replication}

Remarkably, in most if not all these reflections, the concepts of reproduction and replication are used as if interchangeable. This explains why the central constraint proposed as the cornerstone of ageing processes is Orgel's error catastrophe (or sometimes Muller's ratchet). The central concept is that, because material objects and processes cannot stay constant for ever, they need to be maintained, repaired or replicated as identical entities replacing the aged ones. The question, then, is to calculate the relative contribution of the error-producing processes and the corresponding repair and maintenance mechanisms, so that one may evaluate the window of opportunity where immortality will counterbalance processes of degradation (Orgel 1970; Kirkwood and Holliday 1975, 1979). The idea that there can be improvement over time rather than decay or stability, as witnessed in reproduction, is not seriously considered.

In a seminal reflection about the constraints operating at the origin of life, where chemical processes could not be as accurate as they are in extant organisms, Freeman Dyson showed that while replication inevitably leads to the accumulation of errors and the error-catastrophe, reproduction is not doomed to lead to progressively less efficient processes, but rather, can progressively improve their efficiency (Dyson 1985). At the other extreme of chemical processes that resulted in life, a similar distinction has also been considered in general reflections about the existence or role of ageing in life history strategies of a most recent domain of life, that of multicellular organisms (Stearns 1992). Although highly relevant to the conjectures detailed here, and in order to make the question simpler, I shall not explore the latter in depth, but, rather, explore the fate of single cell organisms (see however some comments at the end of this essay). Rather than discuss ageing using multicellular organisms as paradigms, I accept the recent experimental data that demonstrate that bacteria age and take a slightly different stance, considering the way components in the cell age, with single-cell bacteria as reference organisms to see whether and how this relates to the way genomes are organised. I explore the reproduction/replication dialogue, basing my reflection on a deep analysis of the structure of bacterial genomes and see how it is related to the process of ageing at the molecular level.

The core of the model constructed by Dyson rests on the demonstration that reproduction predates replication and can improve over time. The model however does not propose explicit implementation of any concrete process (Dyson 1985). It shows mathematically how prebiotic metabolic systems could progressively become more and more accurate, before they could discover replication. Interestingly, this approach is conceptually somewhat similar to the viewpoint taken in the disposable soma theory, with a completely different background and aim. The argument I wish to develop here is to show how the creation of a link between these views and information theories can lead to very concreteexperimentally testable-hypotheses.

Making young entities from aged ones implies creating (or recovering) some information. Despite the sociological separation between the domains of Physics and Biology the latter rests on all the constraints imposed by the former. It is therefore natural to place Biology in the context of recent developments in Physics to explore whether this would help getting deep insights into deep questions of Biology, while proposing experiments that would lead us to better understanding. Before getting into the heart of the matter, we thus need to revisit some of the concepts of Information Theory. This is particularly fit when considering genomes from the algorithmic point of view that underlies the function of the genetic program. 


\section{Creation of information is reversible}

Information is a deep concept, typical of what the mathematician John Myhill named a «prospective character», i.e. a concept that becomes deeper and deeper as it is further discussed (Myhill 1952). Since its first formalisation in the theory of communication devised by Shannon (Shannon and Weaver 1949), the depth of the concept has been progressively increased in the case of sequences of symbols, typical of what we find in genomes (Cover and Thomas 1991; Yockey 1992; Danchin 1996, 2003). For a long time, it was intuitively assumed that creation of information required energy (typically, according to Leo Szilard, creation of one bit of information would require $1 / 2 \mathrm{kT}$ in energy (Szilard 1929)). As a natural outcome of this intuition, Rolf Landauer, working at IBM on the integration of logical gates into what was to become microprocessors, asked the question whether this would lead to unbearable heat in computing devices, which were meant to become smaller and smaller, while computation kept becoming faster and faster as technology kept improving. In a seminal article in 1961, curiously overlooked, he showed that creation of information, contrary to the accepted view of the time, was reversible and therefore did not involve energy consumption (Landauer 1961). Together with Charles Bennett, he later completed the theory. The overall outcome was that creation of information is reversible, but that erasing information from the memory where all the intermediary steps are collected requires energy, typically of the amount predicted by Szilard (Bennett 1988). In short, creation of information can occur in physical systems, and does not contradict any principle in physics. However, Landauer and later Bennett remarked that information is created among a large number of states that occupy a huge number of space or energy states, or are extremely slow. When considering the production of information, therefore, the question is not whether it is possible, but how it can be recovered from an ocean of noninformative states.

With this understanding, evolution of living organisms (and of any other physico-chemical process where there is accumulation of information) can be understood as the question of telling which, among a variety of individual elements at a given time, must be retained for further evolution. Put otherwise: considering creation of information as an authentic principle of physics, what is the associated principle that makes emerge the entities that have been accumulating information? Because this process requires telling some entities from the bulk, it is a screening process. Because this process requires making room for these entities to accumulate, it is a selective process. Hence, the physics of any system producing information cannot work without a process of selection. In a nutshell, presented in this way, Natural Selection is an authentic principle of physics. It must therefore obey specific rules that need to be formalised.

\section{Natural Selection as a novel but straightforward principle of physics}

This being said - and this rests on the demonstration by Landauer and Bennett that creation of information is reversible-it becomes essential to understand the principles of how Natural Selection-a concept of Biology—works in physical terms. Several ideas can be put forward. I develop here the main directions along which at least one pathway works, that seems to operate at many levels in biological systems, and I illustrate it in the case of bacteria. The basic idea is to follow the second part of the Landauer-Bennett theorem, that which states that erasing memory requires energy, and to look for processes that use energy in an intuitively unexpected way in order to make the relevant ones stand out.

Creation of information requires many steps, it starts from a given complex of dynamic interacting entities, that progressively transforms into variants, among which some have a higher information than that of the original complex. (Some of) those (these entities comprise physical objects, but also processes, such as metabolic fluxes) which are not relevant in terms of increase in information need to be destroyed to make room for those which have a higher information. Within this conceptual frame, this destruction process-Natural Selection-cannot simply be identified with ageing or weathering followed by decay and degradation. It must, at least from time to time, actively discriminate between entities that are in some degree functional and those that cannot function. This is because the process needs to avoid destroying the elements that carry an increased 
information, and this is where energy comes in. Energy has to be consumed to make innovations stand out, in a discriminant process: energy is used to prevent degradation of functional entities, permitting destruction of the non-functional ones (see below).

We note that with this view Natural Selection cannot be purely random or neutral (Kimura and Ota 1974). In other words, simple housekeeping degradative systems will not be sufficient to have the role expected from Natural Selection. Because it requires energy to operate, it can retain or recruit functional entities: this explains part of the controversy surrounding the concept, which is usually assumed to be purely passive (see however the widely spread Spencerian "selection of the fittest" (Spencer 1864), which assumes implicitly some mechanism permitting comparison between fit and unfit organisms). Deep implications of the present contention cannot be discussed in this short essay. It can be noticed however that Kimura himself is aware of the difficulties inherent to a purely neutral selection process (see (Azencott and Ruget 1977)), as he introduced an active selective parameter to take this requirement into account (Kimura 1979). Indeed, it can be shown that purely blind destruction would not work in the long term in a finite population because it would erase both uninformative and informative objects or processes. To understand Natural Selection we must therefore consider specifically the destructive (selective!) steps that are associated to energy consumption and identify, among these, those which are relevant to information.

Interestingly, and in agreement with this line of reasoning, many processes pertaining to improvement of the quality of information are pervasive in molecular biology processes. Noting that a straightforward application of the inevitable noise created by thermal fluctuations at $300 \mathrm{~K}$ (the temperature where living organisms thrive), would lead to an unbearable error rate in the synthesis of proteins, John Hopfield (Hopfield 1974) and Jacques Ninio (Ninio 1975) analysed the essential step of translation of messenger RNAs into proteins along lines similar to those that would draw aside the hypothesis of strict neutralism. They proposed the following idea to account for the remarkable accuracy of the translation process (deciphering the genetic code without an energy-requiring selective step would lead to an error rate resulting in synthesis of essentially aberrant proteins): there is proof-reading steps that tell whether a correct amino acid is loaded on tRNA and a correct codon is deciphered, and that discard the erroneous objects from their target interactions. These essential steps use energy, via ATP and GTP hydrolysis. The basic idea is simple, as a thought experiment illustrates (Gueron 1978): in a pool where we have both black and red fishes swimming together, we wish to catch (almost) exclusively red fishes, knowing that, when in a net with holes having a shape of the size of the fish, but asking for some effort to go through, black fishes react very fast and succeed in getting out, while the red fishes are much slower in that escape. The idea is to use a net, catch fishes (equally black and red) and wait with the net suspended above the pool for some time (not too short, not too long), and then place it on the bank, recovering fishes: most will be red fishes. This is clearly a selective energy-consuming, information-gaining procedure.

This idea can be generalised as follows. A biological system expresses particular objects (RNAs, proteins, metabolites) and processes (metabolic pathways) some of which are accurate, some of which are variants, in particular, as they age as time elapses. Despite this lack of exactitude, an aged system will generally be able to express metabolic pathways and generate young systems, thereby making new objects using old objects (Aguilaniu et al. 2003). Part of the aged objects can be discarded by a variety of degradative processes (Terman et al. 2007) or disposed of in "garbage" structures (Aguilaniu et al. 2003; Stroikin et al. 2005), but the system nevertheless needs to be able to sort out those objects or processes which are functioning best. We assume that this specific type of selection requires energy, noting that if this assumption is correct, then we need to identify the genes coding for the corresponding objects. In short, as implicitly recognised by Dyson when modelling the first steps of the origin of life (Dyson 1985), reproduction can increase the information carried by the system. This increase comes as individual steps. Accumulation of information requires a ratchet-like process that will progressively accumulate accurate objects in a collection of objects that, for that particular purpose, can be considered «junk», but are not «waste». This requires that room is made to permit this accumulation. Making room asks for destruction of some of the objects that occupy the corresponding space, but not in a strictly 
random way as accurate objects need to be preserved from destruction. Again, random destruction would destroy accurate and inaccurate objects equally well, and would not, in general, result in accumulation of accurate objects.

Hence, and this is the most important feature of the present conjecture, that differs from previously proposed models usually meant to propose scenarios for the origin of life, there is a need for a process that, using energy, selects out of the mixture those objects that should preferably not be destroyed. The difference between accurate and inaccurate entities is that the former can be engaged in forming complexes or in metabolic processes. We postulate that when this happens, the entity (process or object) has featurespossibly a particular metabolic flux, possibly rigidity, possibly specific conformation features such as a specific proline isomer, absence of isoaspartates in proteins, etc. - that can be identified by the degradative machinery. Using a source of energy these entities could be rejected by the machinery, and therefore remain intact. By contrast, entities that are not engaged in a complex or a metabolic process would be degraded. This is an exact counterpart of the Hopfield-Ninio kinetic proof-reading process in macromolecular biosyntheses. We must note also that this means that degradative systems are of two types. Some do not require energy for functioning, some require energy; only the latter are directly used in the process of creation of information, the only process that can lead to immortality. This is consistent with the role of mitosis in somatic cell divisions and fits with the idea that cell multiplication dilutes out damaged objects (Brunk and Terman 2002), with a qualification: dilution, per se, would not be enough to permit immortalisation because of the inevitable thermal fluctuations that lead to large deviations from the mean (Azencott and Ruget 1977).

\section{Comparative bacterial genome organisation}

The proposed view assumes that separating between reproduction and replication permits information to be accumulated in a ratchet-like process. Until now this model is presented in an abstract way. It is therefore essential to see whether we can identify in bacterial genomes the (presumably ubiquitous) functions that would explicitly be related to these hypothetical processes. More precisely, we need to identify among those the functions which would code for the energy-dependent processes we have postulated. In this endeavour we benefit from the huge number of genome sequences that are now available. However, a practical difficulty prevents the obvious straightforward comparison between all genomes, looking for genes which would be ubiquitous. Indeed, functions can be fulfilled by different objects and ubiquitous functions are often expressed via gene products of different origins: acquisitive evolution is pervasive in biology (Thompson and Krawiec 1983; Ashida et al. 2005). Hence, even if we expect some functions to be ubiquitous, we do not expect that the genes of interest will be strictly ubiquitous. Fortunately, because organisms derived from each other via an evolutionary process, when an object has been selected in performing a function, it tends to persist through generations, allowing us to identify "persistent" genes, i.e. genes that are present in a clique of genomes but not necessarily in all of them (Fang et al. 2005). Subsequently, knowing these genes permits us to identify (most of) the ubiquitous functions we need to consider as essential to life and to identify the corresponding genes when they do not belong to the persistent set (see an example in (Mechold et al. 2007)).

Analysis of a large number of bacterial genomes allowed us to construct a set of persistent genes and of their conserved syntenies. A detailed analysis of conservation of proximity of genes in genomes revealed a remarkable feature of their organisation: both persistent genes and rare genes tend to stay clustered together (Danchin et al. 2007), making two highly consistent families of genes, separated by a large twilight zone (Fig. 1). Remarkably, the connection network of the persistent genes is reminiscent of a scenario of the origin of life, suggesting "paleome" (from $\pi \alpha \lambda \alpha 1 o \varsigma$, ancient) for its name, as it recapitulates the three phases of a scenario based on surface metabolism (Danchin et al. 2007): synthesis of small molecules on solid surfaces (including ribonucleotides and co-enzymes), substitution of solid particle surfaces by an RNA-world where transfer RNA played a central role, and invention of template-mediated information transfer (Danchin 1989). The paleome is made of approximately 500 genes, that both tend to persist in genomes and persist in the way they cluster in genomes. 
This gene set is made of two approximately equivalent subsets, that differ in the way they can be inactivated with or without loss of capacity to result in colony formation on plates. Essential genes (which cannot be inactivated without loss of capacity to live) contribute to the construction of the cell and to replication of its genome. They make approximately
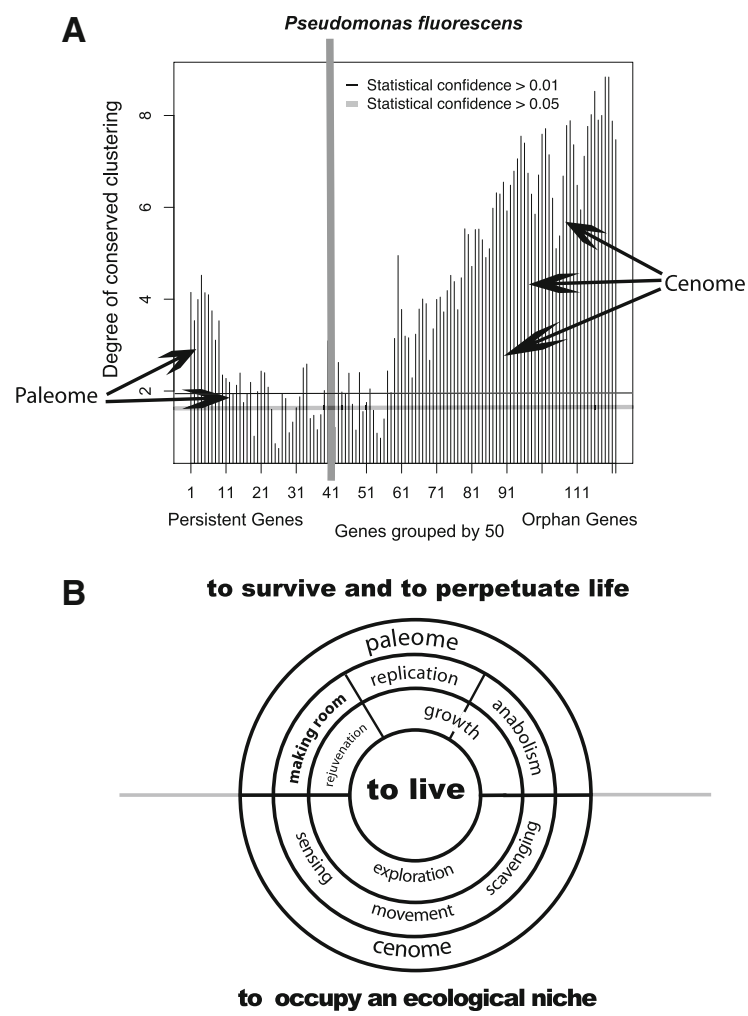

Fig. 1 Gene clustering in a bacterium with a large genome, Pseudomonas fluorescens, as a function of their frequency in bacterial genomes (redrawn and modified after supplementary figure 1, 118 (Danchin et al. 2007)). (a) On the abscissa, genes are grouped by clusters of 50 genes, as a function of their frequency in available bacterial genomes longer than 1,500 genes: the leftmost groups are present in most if not all genomes, and this number progressively decreases along the abscissa, with the group of 50 genes present on the rightmost bars present only in $P$. fluorescens. On the ordinate is represented the tendency for the genes in each group of 50 genes to remain clustered together in the genomes where they are present. The grouping of the genes on the left, making the paleome, is reminiscent of a scenario of the origin of life, while the genes on the right, making the genome, permit cells to occupy a particular niche (Danchin 2007). (b) The genes in the paleome make two categories, persistent essential genes and persistent non-essential genes (Fang et al. 2005). The latter category codes for proteins that use energy to maintain and repair the cell functions as well as genes involved in managing energy involving polyphosphates half of the paleome. The complement is made of nonessential persistent genes. They are involved in functions that are generally annotated as related to maintenance and repair as well as to some specific metabolic pathways (Fang et al. 2005). This latter class is not strictly essential, as the corresponding genes can be inactivated without total loss of viability (Kobayashi et al. 2003; Baba et al. 2006). Analysis of the paleome therefore suggests that we should separate between two different processes, both essential in the long term but with different contribution to essentiality in the short term: the latter category of genes appears to contribute to perpetuation of life rather than to permit life per se. Preliminary experiments are indeed consistent with this view: the plating efficiency of strains inactivated in genes of the second category is rapidly decreasing over time.

By contrast, the set of genes acquired by horizontal gene transfer corresponded to genes important for the cell to survive in a particular environmental niche, not to provide the basal functions for life. This very large class, which we do not consider further here, tends to comprise new members in different strains of the same species. It has accordingly been named the cenome (Danchin 2007), to make reference to its role in permitting the organism to live in a particular niche (after kolvos, common, biocenose is a common concept in ecology, created by Karl Möbius in 1877, see e.g. (Movila et al. 2006)).

\section{Producing new information via energy-dependent degradation processes}

The information accumulating process I considered has to be very effective, and to result in an information-rich outcome in a fairly large number of situations. To understand selective stabilisation of novel information during reproduction, we must therefore consider specific destructive steps that are associated to an energy-consuming step and identify, among these, those which are relevant. This implies that specific processes are implemented to put into action the metabolic capacity of the organism. A central question we need to answer is identification of core metabolic processes that are used to permit selection of young structures in an aged organism. Can we find them into the non-essential part of the paleome? 
At this point of our reasoning, we find that we possess two elements in our conjecture which may be related to each other and may help us to identify the missing elements. On the one hand, we need to find out genes that correspond to the energy-dependent processes necessary to accumulate information in a ratchet-like mechanism during the reproduction phase of the life of the organism. On the other hand we have a set of persistent genes, corresponding apparently to ubiquitous functions (for example, this is the case of the degradation of very short RNAs, an essential step coded by different proteins in different bacterial clades (Mechold et al. 2007)), to which we could not easily ascribe an essential role in the cell.

An analysis of this latter class of genes shows us that, indeed, energy is involved in many of their functions. In particular, we find that many of the enzymes of the "degradosome", essential for RNA degradation, belong to this paleome genes category (for example, the core of the degradosome, polynucleotide phosphorylase, which uses phosphorolysis to produce nucleoside diphosphates-energy-rich compounds-is indeed non-essential (Portier 1980)). While this structure has explicitly been identified in E. coli, where it is associated to polynucleotide phosphorylase and enolase, both energy compliant functions (Carpousis 2007), the counterpart has not yet been identified whether in Firmicutes or in Eukarya, for example. However, a thorough review of the literature, associated to a general analysis of the co-evolution of genes in B. subtilis suggests that, at least in the former case, a degradosome-like structure, directly associated to energy-producing enzymes of glycolysis, is also likely to exist in Firmicutes, made of several components that differ from those present in gamma-Proteobacteria (Danchin 2008). In the case of Eukarya, finally, the exosome is also tightly associated not to hydrolysis, but to RNA phosphorolysis, which is an energysaving process (Lin-Chao et al. 2007). In parallel, genes coding for ATP-dependent proteases as well as ATP-dependent RNA helicases belong to the paleome. Taken together, these functional associations are remarkably consistent with the present conjecture.

A central question remains, however. That of the origin of the energy source that will be used for the information preserving processes. Here again, analysis of the paleome genes provide us with an extremely interesting hypothesis. Indeed, among non-essential paleome genes are the genes that metabolise polyphosphate $(\operatorname{poly}(\mathrm{P}))$. Generally assumed to play the role of phosphate storage, poly $(\mathrm{P})$, a ubiquitous potential source of energy, is systematically associated to RNA degradation and in particular to the $E$. coli degradosome (Blum et al. 1997), as well as to the predicted homologous structure found in Firmicutes (Danchin 2008). Poly(P) has been detected in various amounts in all organisms tested to-date (Brown and Kornberg 2004). The detailed pathways of its metabolism are however still very poorly known, despite the extensive work of the late Arthur Kornberg and his coworkers who unravelled many of the remarkable features of poly(P)-deficient strains (Fraley et al. 2007). Three poly(P) biosynthetic pathways are known, but they apparently fail to account for the bulk of poly(P) accumulation. The major enzyme of poly(P) metabolism, polyphosphate kinase (PPK) catalyzes reversibly and processively phospho-transfer between ATP and poly(P). Another class of processive polyphosphate kinases, PPK2, prefers GTP and is widely conserved in bacteria (Zhang et al. 2002). Many organisms have both PPK and PPK2 in their genomes, a few have only PPK2, some only PPK, and many have neither PPK nor PPK2. The latter have however enzymes, namely PpnKA (YjbN) and PpnKB (YtdI), involved in the poly $(\mathrm{P})$-dependent biosynthesis of NADP (the activity has been biochemically established in Mycobacterium tuberculosis (Raffaelli et al. 2004)) which could be coding for the missing (or a subunit of the missing) polyphosphate kinase. In $B$. subtilis ppnKA is an essential gene in synteny with several genes involved in RNA metabolism or related activities (Danchin 2008).

When cells are ageing, under circumstances when nutrient supplies are limiting for example, the energycharge of the cell will be depleted. In particular, it cannot be expected that ATP is stable enough to maintain its concentration over a long period of time. Under such circumstances a mineral would be particularly stable, and this may well account for the ubiquity of poly $(\mathrm{P})$. The role of this mineral has not yet been explored in this light, although Brown and Kornberg proposed that it might have had an important role at the origin of life (Brown and Kornberg 2004). Furthermore, it seems remarkable that some of its properties would be extremely fit with the present conjecture. Indeed, for example, 
some biochemical assays of adenylate kinase can use poly $(\mathrm{P})$ as the energy-rich phosphate bond instead of ATP (Ishige and Noguchi 2001). In the same way, the second step in the formation of nucleoside triphosphates involves nucleotide diphosphokinase: it can also use poly $(\mathrm{P})$ as a energy-rich phosphate donor, restoring easily the ATP and GTP pools (Ishige et al. 2002). Associated to the phosphorolysis of RNA (Danchin 2008), it seems therefore quite likely that an aged cell will be able to restore its energy capacity well before it can make its core metabolism based on electron transfers work. In this respect it may also be revealing that NAD kinase may use poly $(\mathrm{P})$ as a phosphate donor (Raffaelli et al. 2004), as NADP is the major electron transfer coenzyme directly associated to anabolism, i.e. construction of the cell. Poly $(\mathrm{P})$ has also been shown to provide the energy source for the Lon protease in E.coli (Nomura et al. 2004). One of the intent of this short essay is to convince investigators that it might be important and extremely interesting to explore further this putative role of poly $(\mathrm{P})$.

Finally, I can venture to propose a further exploratory conjecture. Poly $(\mathrm{P})$, by its very structure, is a strong calcium binding mineral. It is present in all cells, and if it has the role we attribute to it, it may also have recruited other functions, as this is very frequently witnessed in evolution. An obvious one, because it is a mineral, is that it could play a role in the structural architecture, the scaffold or casing of organisms. This may have been at the origin of bones in vertebrates. Why not think, then, that bones could still have some of the initial function of poly $(\mathrm{P})$ as an energy source used against ageing processes? This might even be at the root of osteoporosis, linking the present conjecture about ageing in bacteria with ageing in vertebrates. Only deep epidemiological studies associated to genetic studies may tell whether this is a valid conjecture or sheer nonsense (Livshits 2005).

\section{Provisional conclusion}

Often taking these processes as interchangeable (and using both words as equivalent), general reflections on life or ageing have favoured to emphasise replication, not reproduction in the process of cell multiplication (Kirkwood 1977; Szathmary 2000, 2006; Brosh and Bohr 2007; Burhans and Weinberger
2007). This led to a particular interpretation of the important processes of maintenance and repair, which certainly play a major role in sustaining the life of individuals as meant to preserve as long as possible their integrity. In the case of higher organisms, deeper reflections however, in particular those on the disposable soma theory (Kirkwood and Holliday 1979), on the mitochondrial-lysosomal axis (Brunk and Terman 2002), and on the evolution of life histories (Stearns 1992), emphasised the role of reproduction as separated from that of replication, and showed that the functions of maintenance and repair had to be conceptually separated from those involved in reproduction.

The most common views are based on the Spencerian interpretation of Darwinism as «selection of the fittest», where maintenance and repair are systematically associated to competitive processes permitting extension of life span against fertility, as the limited amount of resources that can be devoted to counteract ageing, will jeopardise the same limited resources that can be devoted to fertility (Kirkwood 1977; Holliday 2006). Except in conditions where analyses deal with questions relating to the origin of life or with the error catastrophe, most of these reflections are based on ageing processes involving multicellular organisms. I have tried here, using the recent demonstration that bacteria age and therefore that aged entities must produce young ones, to explore the duality reproduction/replication and to relate it to the increasing knowledge of bacterial genome organisation that we derive from comparative genomics.

This view, as the one that several decades ago proposed the disposable soma theory but following a different path, goes far beyond the molecular processes permitting maintenance and repair. It captures their essence along a further line, the fact that in many occasions they are poised to increase the information content of the entities (physical objects and dynamic processes) that are generally simply interpreted as being repaired. I have tried to show here that considering the remarkable properties of information management in material systems permits us to consider living organisms as poised to accumulate information in a ratchet-like manner. As in the disposable soma theory, the ultimate outcome of this process is to escape the inevitability of ageing, not by perpetuating the individual but by systematically 
creating a progeny made of young organisms. In some instances, this uses processes accumulating aged structures in the mother cell, as in Saccharomyces cerevisiae (Aguilaniu et al. 2003). In other cases, such as rapidly dividing bacteria, the aged structures concentrate at the poles, progressively leading to aged cells which will die, while young cells will continue to multiply (Lindner et al. 2008). Even in multicellular organisms, where it was thought that mitosis resulted in identical daughter cells, it is now established that proteins specifically targeted for proteasomal degradation are inherited preferentially by one mitotic daughter during somatic cell division (Fuentealba et al. 2008).

This process, which accumulates information in at least one member of the cell's progeny has the considerable advantage that, at the same time, it is setting the stage for accumulation of any type of information that may have been created. The consequence is that this may occasionally result in evolution of progressively information-richer cells. In this context, it becomes interesting to entirely reappraise the discovery of "adaptive mutations", which caused much controversy 20 years ago because they were (wrongly) interpreted as suggesting Lamarckian evolution (Cairns et al. 1988; Danchin 1988). Indeed, the core of the reflection proposed here is the idea that, in order to accumulate information, there is a need for an energy-dependent process of «making room» for young or novel entities that will replace the used or aged ones. It could well be, then, that at least some of the genes of the nonessential paleomes are involved in this process. We have constructed a particular E. coli strain that permits easy identification of adaptive mutations (Danchin 1993 (2007)), and we are in the process of investigating whether inactivation of some of the relevant genes prevents their creation. Success in this domain would be a significant proof of concept of the conjecture proposed here, where life and perpetuation of life being considered as separate processes, the latter being able to capture innovation.

Basing the reasoning on Information Theory, I see Natural Selection as this very process, which therefore becomes an authentic principle of Physics. To my view, this is particularly remarkable, and may not be a coincidence (historically, related ideas tend to appear simultaneously everywhere), at a moment when classical physics and quantum physics are getting reconciled via a renewed view of Information Theory. As noted by Steane: «Historically, much of fundamental physics has been concerned with discovering the fundamental particles of nature and the equations which describe their motions and interactions. It now appears that a different programme may be equally important: to discover the ways that nature allows, and prevents, information to be expressed and manipulated, rather than particles to move» (Steane 1998). I wish to extend this view to the realm of Biology.

As a follow up of the observation that replication could not exist at the very origin of life as this would have required some kind of external intervention (Dyson 1985; Szathmary 2006), I have explored here, in the case of bacteria, the consequences of a reproduction process that would be able to create and retain newly created information, in particular. I have established that a system submitted to the trio variation/selection/amplification can accumulate information (within material objects or dynamic processes) when the selection process consumes energy in a step discriminating between stable and less stable interactions. Rather than simply derive a conceptual justification, as this has been repeatedly proposed under a variety of philosophical postulates (see one example of a recent discussion in (Sterelny 2001)), I suggested a concrete justification that may be submitted to experimental falsification. Because it is based on the «measurement» of the quality of an interaction (via its stability) this process will progressively result in the construction of systems accumulating interactions, typical of what is witnessed in the evolution of living organisms. A noteworthy consequence of this observation is that it is not a paradox that there is a general tendency for some branches of life to evolve, without predictable directions, toward progressively more elaborate forms (discovery of multicellularity was one of those remarkable events). We may further note that this process spans domains of Physics and Chemistry much larger than simply the extant living organisms, and that it may apply, with appropriate qualification, to the question of the origin of life in a view that complements and renovates Dyson's reflection. In the domain of Sociology, amusingly, it accounts for the frequent polemics triggered after a discovery has been made. Typically, investigators challenge that somebody made a discovery, saying "I wrote and discussed the same thing; I am therefore a 
co-discoverer." Not so, in general. Why? Indeed the challenger wrote and said things that corresponded to the discovery, but (s)he also said many other things in parallel. The difference with the discoverer is that the latter "made room", eliminating the ideas other than that of the discovery, and this required a process creating information.

At this point of the analysis, emphasis is placed on ageing, via the fact that reproducing metabolic processes can rejuvenate organisms via a selective energy-dependent catabolism that makes room for young functional objects by destructing old ones without affecting functional ones. It is therefore a reflection that is distinct from the prevalent view that ageing is a non-adaptive process that escapes the force of Natural Selection. Or, rather, this view would tend to separate the consequences of ageing in two different categories. In some cases, such as the situation illustrated in the disposable soma theory (which is valid not only for multicellular organisms, but also for budding yeast) the aged individual plays the role of the rubbish bin, that collects nonfunctional entities and all innovation is in the progeny (when it exists) (Stroikin et al. 2005). In other cases, as in pseudo-symmetrically dividing cells, the separation between aged and young entities is much less perfect and leads to progressive loss of viability (Stewart et al. 2005). In this case there is much room for capturing innovation, as this can happen in the course of multiple generations. I conjecture that this might be the explanation of the existence of "adaptive mutation" repeatedly discovered in bacteria. In multicellular organisms, this process is typically developing at mitosis (Fuentealba et al. 2008). This has the remarkable consequence that a phenomenon similar to that of adaptive mutations could permit stem cells to uncover pathways leading to their immortalisation. The implementation of the process we conjecture requires an integrated ensemble of objects, typically those coded by the persistent gene set in bacterial genomes, the paleome. I have identified some of the relevant genes of the paleome and conjectured that a major source of energy involved in the maintenance system could be polyphosphate, a mineral that is ubiquitous in living cells. At this point of the reasoning we understand how an old cell, using energy, can renovate its machinery. A strong prediction of the present model is that the onset of the replication process should be delayed during this renovation step. Indeed, by contrast with reproduction, replication is strongly sensitive to the error catastrophe because of the recursive way it is put into action (Orgel 1963, 1970; Kirkwood and Holliday 1975; Kirkwood 1977; Dyson 1985). It will be interesting to identify the counterparts of the paleome genes in eukaryotes, and to see how their role (including metabolism of polyphosphate) could be related to the onset of cancer.

Acknowledgements I wish to dedicate this work to the memory of Rolf Landauer (1927-1999). I thank Moshe Yaniv and anonymous reviewers for their constructive comments and Eric Fourmentin for his continuous interest and support. This work has been supported by the PROBACTYS programme, grant CT-2006-029104 in an effort to define genes essential for the construction of a synthetic cell and the BioSapiens programme, grant LSHG CT-2003-503265.

Open Access This article is distributed under the terms of the Creative Commons Attribution Noncommercial License which permits any noncommercial use, distribution, and reproduction in any medium, provided the original author(s) and source are credited.

\section{References}

Aguilaniu H, Gustafsson L, Rigoulet M et al (2003) Asymmetric inheritance of oxidatively damaged proteins during cytokinesis. Science 299:1751-1753. doi:10.1126/science. 1080418

Ashida H, Danchin A, Yokota A (2005) Was photosynthetic $\mathrm{RuBisCO}$ recruited by acquisitive evolution from RuBisCO-like proteins involved in sulfur metabolism? Res Microbiol 156:611-618. doi:10.1016/j.resmic.2005.01.014

Azencott R, Ruget G (1977) Mélanges d'équations différentielles et grands écarts à la loi des grands nombres. Zeitung Wahrscheinlichtkeitstheorie verw. Gebiete 38:1-54. doi: 10.1007/BF00534169

Baba T, Ara T, Hasegawa M et al (2006) Construction of Escherichia coli K-12 in-frame, single-gene knockout mutants: the Keio collection. Mol Syst Biol 2, 20060008

Bennett C (1988) Notes on the history of reversible computation. IBM J Res Develop 44:270-277

Blum E, Py B, Carpousis AJ et al (1997) Polyphosphate kinase is a component of the Escherichia coli RNA degradosome. Mol Microbiol 26:387-398. doi:10.1046/j.13652958.1997.5901947.x

Brosh RM Jr, Bohr VA (2007) Human premature aging, DNA repair and RecQ helicases. Nucleic Acids Res 35:75277544. doi:10.1093/nar/gkm1008

Brown MR, Kornberg A (2004) Inorganic polyphosphate in the origin and survival of species. Proc Natl Acad Sci USA 101:16085-16087. doi:10.1073/pnas.0406909101

Brunk UT, Terman A (2002) The mitochondrial-lysosomal axis theory of aging: accumulation of damaged mitochondria 
as a result of imperfect autophagocytosis. Eur J Biochem 269:1996-2002. doi:10.1046/j.1432-1033.2002.02869.x

Burhans WC, Weinberger M (2007) DNA replication stress, genome instability and aging. Nucleic Acids Res 35: 7545-7556. doi:10.1093/nar/gkm1059

Cairns J, Overbaugh J, Miller S (1988) The origin of mutants. Nature 335:142-145. doi:10.1038/335142a0

Carpousis AJ (2007) The RNA degradosome of Escherichia coli: an mRNA-degrading machine assembled on RNase E. Annu Rev Microbiol 61:71-87. doi:10.1146/annurev. micro.61.080706.093440

Clarke S (2003) Aging as war between chemical and biochemical processes: protein methylation and the recognition of age-damaged proteins for repair. Ageing Res Rev 2:263-285. doi:10.1016/S1568-1637(03)00011-4

Cover T, Thomas J (1991) Elements of information theory. Wiley, New York

Danchin A (1988) Origin of mutants disputed. Nature 336:527. doi: $10.1038 / 336527 \mathrm{a} 0$

Danchin A (1989) Homeotopic transformation and the origin of translation. Prog Biophys Mol Biol 54:81-86. doi: 10.1016/0079-6107(89)90010-2

Danchin A (1993 (2007)) Bacteria are not lamarckian. HAL arXiv:q-bio.GN/0702032:hal-00130797

Danchin A (1996). On genomes and cosmologies. In: ColladoVides J, Magasanik B, Smith T (eds) Integrative approaches to molecular biology. The MIT Press, Cambridge, USA, p 91-111

Danchin A (2003) The Delphic boat. What genomes tell us. Harvard University Press, Cambridge, MA, USA

Danchin A (2007) Archives or palimpsests? Bacterial genomes unveil a scenario for the origin of life. Biol Theory 2:5261. doi:10.1162/biot.2007.2.1.52

Danchin A (2008) A phylogenetic view of bacterial ribonucleases. Prog Nuc acids Res Mol Biol 83:000-000

Danchin A (2009) Bacteria as computers making computers. FEMS Microbiol Rev 33:000-000

Danchin A, Fang G, Noria S (2007) The extant core bacterial proteome is an archive of the origin of life. Proteomics 7:875-889. doi:10.1002/pmic.200600442

David CL, Keener J, Aswad DW (1999) Isoaspartate in ribosomal protein S11 of Escherichia coli. J Bacteriol 181: 2872-2877

Dyson FJ (1985) Origins of life. Cambridge University Press, Cambridge, UK

Fang G, Rocha E, Danchin A (2005) How essential are nonessential genes? Mol Biol Evol 22:2147-2156. doi: 10.1093/molbev/msi211

Fraley CD, Rashid MH, Lee SS et al (2007) A polyphosphate kinase 1 (ppkl) mutant of Pseudomonas aeruginosa exhibits multiple ultrastructural and functional defects. Proc Natl Acad Sci USA 104:3526-3531. doi:10.1073/ pnas.0609733104

Fuentealba LC, Eivers E, Geissert D et al (2008) Asymmetric mitosis: unequal segregation of proteins destined for degradation. Proc Natl Acad Sci USA 105:7732-7737. doi:10.1073/pnas.0803027105

Galletti P, De Bonis ML, Sorrentino A et al (2007) Accumulation of altered aspartyl residues in erythrocyte proteins from patients with Down's syndrome. FEBS J 274:52635277. doi:10.1111/j.1742-4658.2007.06048.x
Gueron M (1978) Enhanced selectivity of enzymes by kinetic proofreading. Am Sci 66:202-208

Holliday R (2006) Aging is no longer an unsolved problem in biology. Ann N Y Acad Sci 1067:1-9. doi:10.1196/ annals. 1354.002

Hopfield JJ (1974) Kinetic proofreading: a new mechanism for reducing errors in biosynthetic processes requiring high specificity. Proc Natl Acad Sci USA 71:4135-4139. doi: 10.1073/pnas.71.10.4135

Ishige K, Noguchi T (2001) Polyphosphate: AMP phosphotransferase and polyphosphate: ADP phosphotransferase activities of Pseudomonas aeruginosa. Biochem Biophys Res Commun 281:821-826. doi:10.1006/bbrc.2001.4415

Ishige K, Zhang H, Kornberg A (2002) Polyphosphate kinase (PPK2), a potent, polyphosphate-driven generator of GTP. Proc Natl Acad Sci USA 99:16684-16688. doi:10.1073/ pnas.262655299

Jenkins J, Pickersgill R (2001) The architecture of parallel beta-helices and related folds. Prog Biophys Mol Biol 77:111-175. doi:10.1016/S0079-6107(01)00013-X

Kimura M (1979) Model of effectively neutral mutations in which selective constraint is incorporated. Proc Natl Acad Sci USA 76:3440-3444. doi:10.1073/pnas.76.7.3440

Kimura M, Ota T (1974) On some principles governing molecular evolution. Proc Natl Acad Sci USA 71:28482852. doi:10.1073/pnas.71.7.2848

Kirkwood TBL (1977) Evolution of ageing. Nature 270:301304. doi: $10.1038 / 270301 \mathrm{a} 0$

Kirkwood TBL (2005) Understanding the odd science of aging. Cell 120:437-447. doi:10.1016/j.cell.2005.01.027

Kirkwood TBL, Holliday R (1975) Commitment to senescence: a model for the finite and infinite growth of diploid and transformed human fibroblasts in culture. J Theor Biol 53:481-496. doi:10.1016/S0022-5193(75)80018-X

Kirkwood TBL, Holliday R (1979) The evolution of ageing and longevity. Proc R Soc Lond B Biol Sci 205:531-546. doi:10.1098/rspb.1979.0083

Kirkwood TBL, Shanley DP (2005) Food restriction, evolution and ageing. Mech Ageing Dev 126:1011-1016. doi:10.1016/ j.mad.2005.03.021

Kobayashi K, Ehrlich SD, Albertini A et al (2003) Essential Bacillus subtilis genes. Proc Natl Acad Sci USA 100: 4678-4683. doi:10.1073/pnas.0730515100

Koyré A (1973) The astronomical revolution: CopernicusKepler-Borelli. Methuen, London

Lam H, Schofield WB, Jacobs-Wagner C (2006) A landmark protein essential for establishing and perpetuating the polarity of a bacterial cell. Cell 124:1011-1023. doi:10.1016/ j.cell.2005.12.040

Landauer R (1961) Irreversibility and heat generation in the computing process. IBM J Res Develop 3:184-191

Lin-Chao S, Chiou NT, Schuster G (2007) The PNPase, exosome and RNA helicases as the building components of evolutionarily-conserved RNA degradation machines. J Biomed Sci 14:523-532. doi:10.1007/s11373-007-9178-y

Lindner AB, Madden R, Demarez A et al (2008) Asymmetric segregation of protein aggregates is associated with cellular aging and rejuvenation. Proc Natl Acad Sci USA 105:3076-3081. doi:10.1073/pnas.0708931105

Livshits G (2005) Genetic epidemiology of skeletal system aging in apparently healthy human population. Mech 
Ageing Dev 126:269-279. doi:10.1016/j.mad.2004.08. 020

Mechold U, Fang G, Ngo S et al (2007) YtqI from Bacillus subtilis has both oligoribonuclease and pAp-phosphatase activity. Nucleic Acids Res 35:4552-4561. doi:10.1093/ nar/gkm462

Médigue C, Krin E, Pascal G et al (2005) Coping with cold: the genome of the versatile marine Antarctica bacterium Pseudoalteromonas haloplanktis TAC125. Genome Res 15:1325-1335. doi:10.1101/gr.4126905

Movila A, Uspenskaia I, Toderas I et al (2006) Prevalence of Borrelia burgdorferi sensu lato and Coxiella burnetti in ticks collected in different biocenoses in the Republic of Moldova. Int J Med Microbiol 296:172-176. doi:10.1016/ j.ijmm.2006.02.006

Muller H (1932) Some genetic aspects of sex. Am Nat 66:118128. doi: $10.1086 / 280418$

Myhill J (1952) Some philosophical implications of mathematical logic. I. Three classes of ideas. Rev Metaphys 6: 165-198

Ninio J (1975) Kinetic amplification of enzyme discrimination. Biochimie 57:587-595. doi:10.1016/S0300-9084(75)80139-8

Nomura K, Kato J, Takiguchi N, Ohtake H, Kuroda A (2004) Effects of inorganic polyphosphate on the proteolytic and DNA-binding activities of Lon in Escherichia coli. J Biol Chem 279:34406-34410. doi:10.1074/jbc.M404725200

Nystrom T (2003) Conditional senescence in bacteria: death of the immortals. Mol Microbiol 48:17-23. doi:10.1046/j.13652958.2003.03385.x

Nystrom T (2007) A bacterial kind of aging. PLoS Genet 3: e224. doi:10.1371/journal.pgen.0030224

Orgel LE (1963) The maintenance of the accuracy of protein synthesis and its relevance to ageing. Proc Natl Acad Sci USA 49:517-521. doi:10.1073/pnas.49.4.517

Orgel LE (1970) The maintenance of the accuracy of protein synthesis and its relevance to ageing: a correction. Proc Natl Acad Sci USA 67:1476. doi:10.1073/pnas.67.3.1476

Partridge L (2007) Some highlights of research on aging with invertebrates, 2006-2007. Aging Cell 6:595-598. doi: 10.1111/j.1474-9726.2007.00334.x

Portier C (1980) Isolation of a polynucleotide phosphorylase mutant using a kanamycin resistant determinant. Mol Gen Genet 178:343-349. doi:10.1007/BF00270482

Raffaelli N, Finaurini L, Mazzola F et al (2004) Characterization of Mycobacterium tuberculosis NAD kinase: functional analysis of the full-length enzyme by site-directed mutagenesis. Biochemistry 43:7610-7617. doi:10.1021/bi049650w

Rattan SI (1996) Synthesis, modifications, and turnover of proteins during aging. Exp Gerontol 31:33-47. doi:10.1016/ 0531-5565(95)02022-5

Rattan SI (2008) Increased molecular damage and heterogeneity as the basis of aging. Biol Chem 389:267-272. doi: 10.1515/BC. 2008.030

Riley M, Staley JT, Danchin A et al (2008) Genomics of an extreme psychrophile, Psychromonas ingrahamii. BMC Genomics 9:210

Rocha E, Fralick J, Vediyappan G et al (2003) A strand-specific model for chromosome segregation in bacteria. Mol Microbiol 49:895-903. doi:10.1046/j.1365-2958.2003. 03606.x
Schrödinger E (1945) What is life? The physical aspect of the living cell. The Macmillan Company, New York

Shannon C, Weaver W (1949) The mathematical theory of communication. The University of Illinois Press, Urbana, USA

Shimizu T, Matsuoka Y, Shirasawa T (2005) Biological significance of isoaspartate and its repair system. Biol Pharm Bull 28:1590-1596. doi:10.1248/bpb.28.1590

Spencer H (1864) Principles of biology. Williams and Norgate, London

Steane A (1998) Quantum computing. Rep Prog Phys 61:117173. doi:10.1088/0034-4885/61/2/002

Stearns S (1992) The evolution of life histories. Oxford University Press, Oxford

Sterelny K (2001) Dawkins vs. Gould: survival of the fittest. Icon Books, Cambridge, UK

Stewart E, Taddei F (2005) Aging in Escherichia coli: signals in the noise. Bioessays 27:983. doi:10.1002/bies.20295

Stewart EJ, Madden R, Paul G et al (2005) Aging and death in an organism that reproduces by morphologically symmetric division. PLoS Biol 3:e45. doi:10.1371/journal. pbio.0030045

Stroikin Y, Dalen H, Brunk UT et al (2005) Testing the "garbage" accumulation theory of ageing: mitotic activity protects cells from death induced by inhibition of autophagy. Biogerontology 6:39-47. doi:10.1007/s10522004-7382-y

Szathmary E (2000) The evolution of replicators. Philos Trans R Soc Lond B Biol Sci 355:1669-1676. doi:10.1098/rstb. 2000.0730

Szathmary E (2006) The origin of replicators and reproducers. Philos Trans R Soc Lond B Biol Sci 361:1761-1776. doi: 10.1098/rstb.2006.1912

Szilard L (1929) Uber die Entropieverminderung in einem thermodynamischen System be eingriffen intelligenter Wesen. Z Phys 53:840-856. doi:10.1007/BF01341281

Terman A, Gustafsson B, Brunk UT (2007) Autophagy, organelles and ageing. J Pathol 211:134-143. doi:10.1002/ path.2094

Thompson LW, Krawiec S (1983) Acquisitive evolution of ribitol dehydrogenase in Klebsiella pneumoniae. J Bacteriol 154:1027-1031

Watt RM, Wang J, Leong M et al (2007) Visualizing the proteome of Escherichia coli: an efficient and versatile method for labeling chromosomal coding DNA sequences (CDSs) with fluorescent protein genes. Nucleic Acids Res 35:e37. doi:10.1093/nar/gk11158

Yamamoto A, Takagi H, Kitamura D et al (1998) Deficiency in protein L-isoaspartyl methyltransferase results in a fatal progressive epilepsy. J Neurosci 18:2063-2074

Yockey HP (1992) Information theory and molecular biology. Cambridge University Press, Cambridge, UK

Zarivach R, Deng W, Vuckovic M et al (2008) Structural analysis of the essential self-cleaving type III secretion proteins EscU and SpaS. Nature 453:124-127. doi:10.1038/nature 06832

Zhang H, Ishige K, Kornberg A (2002) A polyphosphate kinase (PPK2) widely conserved in bacteria. Proc Natl Acad Sci USA 99:16678-16683. doi:10.1073/pnas.262655199 